\title{
Reprodução assistida como causa de morbidade materna e perinatal
}

\author{
Assisted reproduction as a cause of maternal and perinatal morbidity \\ João Luiz Pinto e Silva ${ }^{1}$, Luis Bahamondes ${ }^{2}$
}

RESUMO

Objetivos: realizar revisão bibliográfica do impacto das técnicas de fertilização assistida e reprodução assistida (RA) na morbidade materna e perinatal. Métodos: foram pesquisadas fontes bibliográficas pelo SCI e MEDLINE, com o intuito de identificar o maior número de estudos relacionados com os termos: fertilização in vitro, reprodução assistida, técnicas reprodutivas, combinadas com morbidade e com mortalidade -materna, perinatal e neonatal. Resultados: a literatura permite concluir que o maior problema de morbidade materna relaciona-se à ocorrência de maior número de gravidezes múltiplas, destacando-se em alguns estudos a maior freqüência de hipertensão induzida pela gravidez e diabetes gestacional. A ocorrência de maior número de gravidezes múltiplas aumenta consideravelmente as complicações maternas, fetais e dos recém-nascidos. Recomenda-se um pré-natal diferenciado, de enfoque multidisciplinar para otimizar resultados. Há evidências de maior número de malformações congênitas. Discutem-se as características especiais deste grupo de mulheres e das diferentes técnicas de RA, particularmente a ICSI, na etiologia dos defeitos congênitos, embora não existam diferenças claras entre os procedimentos. Algumas meta-análises recentes mostraram que o número de malformações congênitas em crianças nascidas por ICSI é maior do que entre as nascidas espontaneamente, mas não mais freqüentes que as nascidas por outras técnicas de RA. Não existe consenso se este fato é decorrente do procedimento per se, da manipulação dos gametas, da indução da ovulação ou do fato de que estes casais são inférteis e do tempo que levam para engravidar. Existem poucos estudos que avaliaram de modo consistente, sistemático e prolongado a evolução perinatal de crianças nascidas mediante a utilização de embriões congelados. Conclusões: em relação às malformações fetais há, definitivamente, maior número entre as originadas de RA, que entre as crianças concebidas naturalmente, com RR de 1,4 a 2,0 (IC 95\%: 1,3-2,7). Não há tempo e registro suficientes para analisar os resultados originados de gravidezes com embriões congelados. Não é claro se os achados devem-se às características dos casais que se submetem a estes procedimentos, ou às peculiariedades de cada método. Muitos dos problemas relacionados à morbidade materna e perinatal devem-se ao significativo número de gravidezes múltiplas originadas de RA.

PALAVRAS-CHAVE: Técnicas reprodutivas assistidas; Fertilização in vitro; Morbidade; Recém-nascidos

\section{ABSTRACT}

Purpose: to carry out a literature review to evaluate the impact of assisted reproductive techniques (ART) on maternal and perinatal morbidity. Methods: specialized data bases such as SCI and MEDLINE were used to identify studies related to the terms: "in vitro fertilization", "assisted reproduction" and "reproductive techniques" in combination with "morbidity", "maternal mortality", "perinatal mortality", and "neonatal mortality". Results: data from published studies allow us to conclude that maternal morbidity is related to an increase in the number of multiple pregnancies. In addition, some studies have reported an increased incidence of pregnancy-induced hypertension and gestational diabetes. Specialized multidisciplinary prenatal care has been recommended to obtain optimal results. An increase in the number of multiple pregnancies considerably increases maternal, fetal and neonatal complications. There is also evidence of an increase in congenital malformations. The particular characteristics of this group of women and the different techniques of assisted reproduction, particularly ICSI, in the etiology of congenital defects were discussed, but no clear differences have been established between the various procedures. Some recent metanalyses show that the number of fetal malformations in infants born as a result of ICSI is greater than in spontaneously conceived infants, but not more frequent than in those born as a result of other ART. There is no consensus regarding whether this fact is a result of the procedure itself, of manipulation of the gametes, ovulation induction, if it is due to the fact that these couples are infertile or a result of the time they take to become pregnant. Few studies have carried out a prolonged, consistent and systematic evaluation of the perinatal evolution of infants born following the use of frozen embryos. Conclusions: with respect to fetal malformations, there is definitely a

Departamento de Tocoginecologia, Faculdade de Ciências Médicas, Universidade Estadual de Campinas - UNICAMP - Campinas (SP) - Brasil.

1 Professor Titular de Obstetricia do Departamento de Tocoginecologia da Faculdade de Ciências Médicas da Universidade Estadual de Campinas UNICAMP - Campinas (SP) - Brasil.

2 Professor Titular de Ginecologia do Departamento de Tocoginecologia da Faculdade de Ciências Médicas da Universidade Estadual de Campinas UNICAMP - Campinas (SP) - Brasil.

Correspondência: João Luiz Pinto e Silva

Caixa Postal 6081 - 13084-970 - Campinas - SP - Telefone: (19) 3788-9567 - Fax: (19) 3788-9304 - e-mail: psilva@unicamp.br 
higher incidence rate among infants born as a result of ART compared to those conceived naturally (RR: $1.4-2.0$; 95\% CI: $1.3-$ 2.7). Insufficient time and data do not yet permit analysis of the outcome of pregnancies resulting from the use of frozen embryos. It is not clear whether these findings are due to the characteristics of the couples who are submitted to these procedures or to the peculiarities of each method. Many of the problems related to maternal and perinatal morbidity are due to the significant number of multiple pregnancies originating from ART. More studies are required in order to clarify these aspects of human reproduction.

KEYWORDS: Reproductive techniques, assisted; Fertilization in vitro; Morbidity; Newborn infants

\section{Introdução}

Vinte e sete anos se passaram desde o nascimento da primeira criança, Louise Brown, por fertilização in vitro (FIV), em 1978. Em 1992 foi introduzida com sucesso a injeção intracitoplasmática de espermatozóides (ICSI) e em 1993 foi informado o nascimento da primeira criança após criopreservação de um embrião. As técnicas de reprodução assistida (RA) têm se aperfeiçoado de modo notável em todo o mundo, e após o primeiro nascimento, milhões de crianças nasceram pelas diferentes modalidades de FIV. A técnica de ICSI, em particular, mudou de forma radical os conceitos anteriores sobre esterilidade e infertilidade, uma vez que permitiu a muitos casais considerados anteriormente como definitivamente estéreis, alcançar possibilidades reais de reverter sua condição.

Apesar do aperfeiçoamento e da expansão do acesso às técnicas de RA, o número de crianças nascidas em todo o mundo ainda é escasso, em comparação aos nascimentos resultantes de fertilização natural. As últimas cifras indicam que para mulheres de até 35 anos a taxa de criança viva ao final do processo é de $28 \%$ por ciclo, e para mulheres de 43 anos ou mais é de apenas $5 \%$ por ciclo $^{1}$. Embora os números sejam dificeis de estabelecer com precisão, estima-se que atualmente em torno de $2 \%$ dos nascimentos no mundo desenvolvido, que em geral mantém registros nacionais de qualidade, ocorram após algum procedimento de RA. Em outros países, especialmente nos assim denominados em desenvolvimento, estes tipos de registros ou são incompletos, inexatos e inconsistentes ou em alguns casos claramente não existem.

Uma das principais preocupações em relação à utilização destes recursos de tratamento é a morbidade que eventualmente possa ocorrer com as mulheres grávidas e suas crianças, tanto a curto quanto a longo prazo. Esta preocupação é crucial nos casos dos nascidos mediante ICSI, já que o único espermatozóide utilizado para a fertilização é obtido diretamente do líquido seminal, nos casos de oligozoospermia, das vesículas seminais, nos de agenesia ou obstrução dos deferentes, e diretamente do parênquima testicular, nos de azoospermia secretória. Nestas situações, o processo natural de fertilização, ou mesmo o artificial durante a FIV, não permite conhecer com eficiência a qualidade da célula masculina, que é escolhida ao acaso, uma vez que, de fato, o que se busca é a transferência de material genético ao interior do óvulo com fins reprodutivos. A manipulação no procedimento poderia afetar, eventualmente, a integridade desse patrimônio, o que justificaria maiores preocupações com a evolução dessas crianças.

\section{Método}

Esta revisão foi organizada por meio de pesquisa de fontes recolhidas na literatura pertinente pelo sistema SCI e MEDLINE com os termos: fertilização in vitro, reprodução assistida, técnicas reprodutivas, combinadas com morbidade e com mortalidade materna, perinatal e neonatal.

\section{Morbidade materna em gravidezes origina- das de procedimentos de reprodução assistida}

Uma das primeiras revelações desta revisão foi a escassez de publicações relacionadas com resultados da evolução da gravidez, morbidade e mortalidade maternas, concentrando-se a maioria bibliográfica nos resultados perinatais e dos recém-nascidos.

A maioria das publicações destaca, de modo enfático, que um dos maiores problemas destas técnicas está relacionado com o maior número de gravidezes múltiplas, duplas e triplas, o que aumenta, consideravelmente, as complicações maternas, fetais e do recém-nascido. Entretanto, nas gravidezes únicas também pode haver problemas. Estudo de coorte multicêntrico, controlado e prospectivo $^{2}$ avaliou, na Alemanha, informações sobre mais de 3.000 gravidezes obtidas por ICSI. Entre as gravidezes únicas, o risco relativo (RR) de complicações obstétricas foi significativamente maior do que entre os casos com gravidez natural usadas como 
controle, para: sangramento vaginal antes e após $28^{\mathrm{a}}$ semana da gravidez (RR 3,8; IC 95\% 2,8-5,2), placenta prévia (RR: 6,4; IC 95\%: 4,0-10,2), oligoidrâmnio (RR: 2,1 ; IC 95\%: $1,5-3,0$ ), insuficiência placentária (RR: 3,7; IC 95\%: 2,7-5,0), descolamento prematuro de placenta (RR 1,8; IC 95\%: 1,2-2,6), trabalho de parto prematuro (RR: 2,6; IC 95\%: 2,3-2,2), anemia (RR: 1,7; IC 95\%: 1,5-1,8), pré-eclâmpsia (RR: 1,3; IC 95\%: 1,1-1,5) e parto prétermo (RR: 1,8; IC 95\%: 1,5-2,1). Quando esses autores avaliaram todas as gravidezes, incluindo as múltiplas, e não somente aquelas com feto único, o excesso de risco em relação ao grupo controle esteve presente para as mesmas complicações, agregando-se somente a incompetência istmo-cervical, devido provavelmente às gravidezes múltiplas em maior número $(23,5$ versus $1,0 \%)$ que se associariam a maior freqüência do problema.

Outra complicação que tem sido associada com os procedimentos de RA é o diabete gestacional. Vários autores têm mostrado que, entre mulheres grávidas por procedimentos de RA, a taxa de diabete gestacional chegou a $21 \%$, sendo significativamente mais alta que nos grupos utilizados como controle (habitualmente partos e gravidezes espontâneas da mesma instituição ou da mesma região geográfica $)^{3,4}$. Neste último estudo, também se identificou, entre mulheres submetidas a FIV, aumento do risco de hipertensão induzida pela gravidez.

Explicação para o risco aumentado para estas complicações poderia ser alterações endometriais e endócrinas reconhecidamente presentes nestas mulheres. Quanto a maior freqüência de incompetência istmo-cervical, poder-se-ia discutir a validade da qualidade do diagnóstico, considerando que na realidade não existiria alteração estrutural dos mecanismos de contenção fetal, congênita ou adquirida, mas apenas incapacidade de impedir o nascimento prematuro por absoluta falta de espaço, no interior da matriz, para as gravidezes de mais de um feto. É digno de registro que os dados de literatura mostram incremento nos casos de parto pré-termo e baixo peso ao nascer entre casais inférteis, independentemente de terem sido submetidos ou não a técnicas de RA ${ }^{5}$. Os resultados indicam que estas gravidezes devem ser sempre acompanhadas em clínicas de pré-natal especializado em assistência de gravidezes de alto risco, com forte enfoque e participação interdisciplinar.

\section{Morbidade perinatal após procedimentos de fertilização in vitro}

A maior quantidade de publicações que envolvem a observação da morbidade de fetos e recém-nascidos, relacionadas com as gravidezes obtidas por FIV, preocupa-se em analisar a ocorrência, tipos e a freqüência de malformações congênitas (MF), se maiores ou menores e com o desenvolvimento psicomotor posterior das crianças.

Estudo francês com 370 crianças nascidas entre 1981 e 1988 em um único centro assistencial, e seguidas até entre 6 e 13 anos de idade, não mostrou diferenças quanto ao desenvolvimento corporal, psicomotor, rendimento escolar, problemas médicos ou cirúrgicos. Também não foi observada freqüência mais alta de $\mathrm{MF}^{6}$ quando comparadas com a população geral.

No entanto, estudo realizado em 1987 na Austrália e Nova Zelândia, com série de 1694 recém-nascidos, concluiu que havia um RR maior para MF, com incidência de 2,2\% (IC 95\%: 1,5-2,9), sendo as mais comuns a espinha bífida (encontrada em 6 crianças quando o esperado era 1,2 caso) e a transposição de grandes vasos (em 4 crianças, para um esperado de 0,6$)^{7}$.

Outro estudo de grande porte, um dos maiores encontrados na literatura, foi o realizado na França com 7024 gravidezes e 6879 crianças nascidas por $\mathrm{FIV}^{8}$. Neste se observou que $26,8 \%$ dos nascimentos foram múltiplos, $29,3 \%$ prematuros e $36,2 \%$ pequenos para a idade gestacional (PIG). Entretanto, o número de MF $(2,8 \%)$ foi similar ao da população em geral $(2,1 \%)$, ao passo que as mortalidades perinatal e neonatal apresentaram taxas maiores que as nacionais.

Resultados similares foram obtidos na Dinamarca ${ }^{9}$, onde o número de $\mathrm{MF}$ foi igual entre crianças de FIV e controles, mas com taxa de prematuridade geral de 23,8\%. Para nascimentos únicos, a taxa de prematuridade foi de $7,3 \%$, semelhante à grande maioria dos países desenvolvidos, sendo $41,2 \%$ para gêmeos e $93,5 \%$ para trigêmeos. Baixo peso ao nascer $(\mathrm{BPN})(<2.500 \mathrm{~g})$ foi observado em $7 \%$ dos nascimentos únicos, em $42,2 \%$ dos gêmeos duplos e $87,1 \%$ para os triplos. Todas estas taxas de BPN foram significativamente mais elevadas que as observadas para a população geral. Muito similares também foram os achados observados na Suécia, onde se avaliaram crianças nascidas entre 1982-1995. A única diferença em relação ao estudo dinamarquês é que o número de $\mathrm{MF}$, entre mais de 5 mil crianças, comparado com mais de 1,5 milhão da população geral, era mais alto (taxa de 5,4\%, com RR de 1,39, IC $95 \%: 1,25-1,54)$, e as taxas de defeitos do tubo neural e atresia de esôfago foram também mais altas que dos controles ${ }^{10}$.

Neste mesmo país, quando se ampliou a revisão até 1997 e o número de crianças ultrapassou 9 mil $^{11}$, o RR de MF continuou maior que o 
esperado $(1,47)$. Este excesso desapareceu, no entanto, quando as taxas foram controladas para variáveis confundidoras tais como: ano de nascimento, idade materna, paridade e tempo de esterilidade. Apesar de haver maior número de MF do tubo neural e esofágicas, este fato já havia sido descrito previamente em gêmeos monozigotos, assim como a ocorrência da síndrome de múltiplas malformações denominada VACTERL, e que poderia ser atribuída a distúrbios precoces do desenvolvimento embrionário ${ }^{12}$. De qualquer forma, convém lembrar que as maiores taxas de gêmeos monozigotos são devidas intrinsecamente aos procedimentos de FIV.

Resultados muito parecidos foram encontrados na Finlândia ${ }^{13}$, com maior mortalidade, prematuridade, BPN, morbidade neonatal e dias de hospitalização, quando as crianças nascidas por FIV foram comparadas a controles. Estes resultados foram atribuídos pelos autores ao maior número de gravidezes múltiplas. Também se encontrou taxa quase quatro vezes maior de malformações cardiacas que nos controles. Não há explicação definitiva para o achado, uma vez que estas MF obedecem a fatores múltiplos como genéticos e ambientais, e menos de $10 \%$ correspondem a aberrações cromossômicas e mutações de um gene único ${ }^{14}$.

Apesar de muitos dos problemas como BPN, prematuridade, morbidade e mortalidade neonatal terem sido atribuídos ao maior número de gravidezes múltiplas, esta interpretação não é totalmente satisfatória. Estudo em Israel ${ }^{15}$ avaliou 104 casais de gêmeos nascidos por FIV comparativamente a 193 gêmeos gerados naturalmente. Observaram que entre os nascidos por FIV houve maior número de nascidos prematuros e maior número de casos com restrição de crescimento fetal e morte fetal. O peso médio ao nascer de ambos os gêmeos foi menor no grupo de FIV. Maior número de neonatos pesaram menos que $1.500 \mathrm{~g}$ ou tiveram Apgar menor que 7 aos cinco minutos, maior número foi admitido em UTI neonatal e entre os segundos gêmeos mais crianças morreram.

Estudo prévio atribuiu à gravidez gemelar com placentas monocoriônicas pior prognóstico que aquela com placentas dicoriônicas ${ }^{16}$. Como as gravidezes múltiplas de FIV são, em geral, formadas por placentas dicoriônicas, raramente monocoriônicas, a interpretação seria que por isso teriam melhor prognóstico. Os dados de Daniel et al. ${ }^{15}$ não apóiam esta suposição, e estes autores acrescentaram a observação de que nestes casos os gêmeos estão expostos a um duplo risco: por um lado por serem gêmeos, e por outro, por haverem sido gerados por FIV.
Outros problemas que devem ser pesquisados cuidadosamente nestas crianças são possivieis seqüelas neurológicas. O estudo realizado na $\mathrm{Su}-$ écia ${ }^{17}$ acompanhou 5.680 crianças nascidas por FIV, comparando cada uma com dois controles, e 2.060 crianças que eram o segundo gêmeo, comparadas também com dois controles que eram segundos gêmeos não gerados por FIV. As crianças nascidas por FIV precisaram com maior freqüência de serviços de reabilitação que os controles (RR 1,7; IC 95\%: 1,3-2,2). O problema neurológico mais comum foi a paralisia cerebral, e as crianças de FIV tiveram risco relativo de 3,7 $(2,0-6,6)$, e a suspeita de atraso no desenvolvimento foi de 4,0 $(1,9-8,3)$. Nas crianças de gravidezes gêmeas não houve risco aumentado em relação aos controles. Aquelas que foram PIG e as prematuras tiveram maiores problemas que as nascidas a termo.

Em informe para o qual foram combinadas informações sobre 27.403 recém-nascidos por FIV na Austrália, França, Estados Unidos e Escandinávia, a taxa global de prevalência para defeitos do tubo neural foi de 1,9. A principal alteração detectada foi a onfalocele, sendo esta taxa maior que a esperada. Não obstante, os dados são conflitantes e devem ser interpretados com cautela, já que há muita diversidade entre os registros ${ }^{18}$. O maior problema com estes resultados é que a indução da ovulação nas mulheres, a prática de procedimentos de FIV e os defeitos do tubo neural são condições médicas raras, o que pode levar a distorção na observação, inclusive por monitorização mais atenciosa destas crianças dado o caráter de serem muito valorizadas por seus progenitores e a equipe médica que realizou o procedimento.

Outro aspecto que tem sido observado é a ocorrência maior de hipospádias entre as crianças masculinas nascidas por FIV. Estudo realizado nos Estados Unidos com cinco anos de avaliação mostrou um RR quase 5 vezes maior para os nascidos por FIV que para os controles (RR: 1,5\% no grupo de FIV vs $0,3 \%$ nos controles). Esta malformação tem sido atribuída também ao uso sistemático, por parte das mulheres tratadas, de progesterona como suporte da fase lútea após a transferência de embriões, mas, as reais causas determinantes da alteração permanecem desconhecidas ${ }^{19}$.

Outras manifestações clínicas pouco estudadas são os problemas oculares em crianças nascidas por FIV, tanto naquelas com capacidade de verbalizar como nos infantes ainda sem capacidade de se comunicar. Anteby et al. ${ }^{20}$ avaliaram 47 crianças nascidas por FIV. Encontraram entre as crianças sem capacidade para se comunicar, por conta da idade, que $15 \%$ tinham visão diminuída, e entre as crianças que já se comunica- 
vam a porcentagem era de $17 \%$. Também houve $22 \%$ de emetropia, $57 \%$ de hipermetropia e $16 \%$ de miopia. Das 47 crianças observadas, 12 (26\%) apresentaram malformações oculares maiores, como doença de Coats, catarata e glaucoma congênitos, hipoplasia do nervo óptico, atrofia óptica idiopática, coloboma com microftalmia e retinoblastoma. Apesar destes achados, não é possivel vincular definitivamente a FIV aos problemas oculares, sendo, entretanto, recomendável que estas crianças sejam examinadas precocemente após o nascimento.

Recentemente, dois estudos, um da Finlândia com os registros nacionais e outro de Iowa, nos Estados Unidos, mostraram que, ainda em gravidezes únicas, o RR de MF está aumentado nas crianças geradas por procedimentos de FIV ou ICSI. Ambos os estudos mostraram aumento das malformações cardiacas e do sistema urogenital e musculoesquelético. Interessantemente, também a hipospádia foi mais prevalente entre estas crianças $^{21,22}$.

\section{Gravidezes com procedimentos de injeção in- tracitoplasmática de espermatozóides}

Esta técnica começou a ser utilizada em 1992, e conseqüentemente, todos os estudos realizados apresentam tempo de seguimento muito curto e ainda insuficiente para uma observação do desenvolvimento destas crianças, sobretudo no que está relacionado ao seu futuro reprodutivo.

Em dois estudos ${ }^{23,24}$ realizados na Bélgica, compararam-se quase 3.000 crianças nascidas por ICSI com número similar nascidas por FIV. Outro estudo do mesmo grupo apresentou informações sobre o seguimento de 1987 crianças oriundas de $\mathrm{ICSI}^{25}$. Houve taxas semelhantes nas duas coortes em relação às gravidezes múltiplas e características maternas. O peso ao nascer, número de complicações neonatais, BPN e mortalidade foram similares. A taxa de MF também foi igual em ambos os grupos, e não esteve, no grupo de ICSI, relacionada com a origem dos espermatozóides utilizados (epidídimo ou testículos), nem com a qualidade dos mesmos. O problema destes estudos é que, embora mostrem que aparentemente ambos os procedimentos são seguros, não houve comparação com a população geral do país estratificada e controlada por variáveis confundidoras como idade materna, paridade, hábitos de uso de tabaco e álcool, entre outras. Outra crítica que se pode fazer é que o tempo de seguimento foi muito curto, de até dois anos. Os autores reconheceram que seria necessário seguimento maior, sobretudo para avaliar desenvolvimento sexual, fertilidade e desenvolvimento psicomotor futuros.
Resultados semelhantes sobre desenvolvimento neurológico foram observados em coorte pequena de crianças nascidas por ICSI comparada com crianças nascidas naturalmente ${ }^{26}$. Estas crianças foram seguidas até os 17 meses de idade, e não houve diferenças inclusive na taxa de MF, apesar de que entre as crianças com pais oligozoospérmicos houvesse maior número de $\mathrm{MF}$, sem que fosse possivel explicação razoável considerando o pequeno número de casos avaliados.

Estudo realizado com 665 crianças nascidas por ICSI na Dinamarca tampouco observou problemas em relação a $\mathrm{MF}$, e os resultados perinatais foram similares aos de outros autores. É importante destacar a possibilidade de viés por não haver comparação dos resultados com outras crianças da população geral ${ }^{27}$.

Entretanto, é importante destacar que tem havido maior quantidade de relatos de taxa de hipospádias, maior que a esperada ${ }^{11,28,29}$, especialmente nas gravidezes obtidas de homens oligozoospérmicos. Uma das possiveis explicações para este tipo de MF, quando se realiza ICSI, é que como estas gravidezes são obtidas, em geral, de homens com defeitos na espermatogênese e anomalias testiculares, provavelmente carreguem algum defeito genético que condicione a transmissão deste tipo de MF. Especula-se que o defeito genético pudesse ser o mesmo fator que provocou a subfertilidade. Entretanto, como foi descrito, também tem sido observado em crianças nascidas por FIV.

Foram avaliadas as anormalidades cromossômicas entre 447 casais compostos por homens e mulheres que iriam participar de um procedimento de ICSI. O número de aberrações encontradas foi superior ao esperado para a população geral, e apesar de que seu número tivesse apenas um risco menor de transmissão aos recémnascidos, a surpresa do estudo foi o alto número de aberrações encontradas entre as mulheres. O achado levou os autores a sugerir que não só os homens, mas também suas parceiras fossem sistematicamente avaliadas do ponto de vista genético, antes de o casal se submeter a um procedimento de $\mathrm{ICSI}^{30}$. Recentemente algumas metaanálises mostraram que o numero de MF em crianças nascidas por ICSI é maior que nas nascidas espontaneamente. Entretanto, não são maiores que naquelas nascidas por FIV. Uma especulação é que o fato de ser infértil, per se, seria suficiente para aumentar o risco de $\mathrm{MF}^{5,31,32}$.

Também tem sido sugerido que haveria aumento de defeitos de imprinting como sindrome de Angelman, em crianças nascidas por FIV ou ICSI, mas ao mesmo tempo se admite que o defeito poderia estar vinculado à subfertilidade dos casais, à superovulação e ao maior tempo para engravidar ${ }^{33}$. 


\section{Gravidezes com embriões congelados}

Existem muito poucos estudos que avaliaram de modo consistente, sistemático e a longo prazo a evolução perinatal de crianças nascidas mediante a utilização de embriões congelados. Em trabalho realizado na França com 82 crianças nascidas entre 1986 e 1994 foram acompanhadas algumas destas crianças até os nove anos de idade, mediante questionários enviados aos pais, que foram respondidos por telefone ou carta, ou pelo pediatra assistente, caso a criança tivesse sido consultada por algum problema de saúde ${ }^{34}$. Para crianças menores de cinco anos o questionário incluiu perguntas sobre o desenvolvimento psicomotor, e para as maiores, foram incluídas perguntas sobre o rendimento escolar em comparação com os outros de sua classe e da mesma idade. Para todos se obtiveram dados sobre peso e altura atuais, e sobre o período perinatal, os dados foram resgatados das histórias clínicas dos hospitais onde se atendeu o parto. Neste grupo houve $8,5 \%$ de gravidezes gêmeas, similar à população geral. A taxa de prematuridade foi de $14,7 \%$ para gravidezes únicas e de $85,7 \%$ para as gêmeas. A taxa de PIG foi de $8 \%$ nas gravidezes únicas e de $28,6 \%$ nos gêmeos, e a de BPN foi de $64,3 \%$ nos gêmeos. Em relação ao seguimento destas crianças observou-se que a altura e o peso foram normais, inclusive para as nascidas prematuras ou PIG. Os problemas médicos clínicos detectados incluíram alergia, asma e refluxo gastroesofágico em alguns casos, assim como os cirúrgicos incluiram hérnia e maior quantidade de cirurgias para adenóides e amígdalas. As MF foram só duas e nenhuma delas maior. O desenvolvimento psicológico, psicomotor e escolar ocorreu dentro do esperado, não havendo, inclusive, repetição do grau escolar atendido.

Outro estudo similar foi realizado na Inglaterra com 91 crianças nascidas de embriões congelados e 83 controles nascidos sem RA, pareados por idade, sexo e classe social. Apesar de que no grupo concebido com embriões congelados houvesse maior taxa de cesárea, menor peso ao nascer e menor idade gestacional, os resultados foram muito similares entre ambos grupos em relação ao desenvolvimento mental avaliado com a escala de Griffiths, modo especial de medir o desenvolvimento mental ${ }^{35}$.

Em relação à incidência de $\mathrm{MF}$ menores, encontrou-se $31,9 \%$ no grupo de embriões congelados e $21,7 \%$ no grupo de controle, sendo principalmente nevus e hemangiomas as mais incidentes. A incidência de MF maiores foi de 3,3 e 2,4\%, respectivamente, sem significado estatístico. As principais foram sindrome de Down, síndrome de
Beckwith-Wiedemann e hipofosfatemia no grupo de embriões criopreservados, e gastroquise e hidronefrose entre os controles ${ }^{36}$.

Apesar de os dados existentes mostrarem risco aumentado para as crianças nascidas depois da transferência de embriões congelados, os estudos são escassos e os poucos casos avaliados não permitem conclusões definitivas. Os estudos com seguimentos pediátricos são prejudicados pelas dificuldades de se utilizarem ferramentas de avaliação padronizadas, pela necessidade de se utilizarem observadores múltiplos, e pela escolha de controles adequados e por insuficiente tamanho amostral. Estes fatos impedem que se possa obter resultados válidos capazes de responder às importantes indagações que devem ser feitas.

Assim se pode concluir que não há estudo adequadamente desenhado e com número suficiente de casos que permitam conclusões válidas. Recomenda-se que se estabeleça um registro multinacional cuidadoso e sistemático, para obteremse dados válidos e consistentes que sirvam médicos e pacientes de elementos eficientes para tomar decisões amparadas por evidências científicas.

\section{A situação no Brasil}

Na Inglaterra, desde 1990 existe uma norma estabelecida pelo Parlamento para regular e vigiar os procedimentos de $\mathrm{FA}^{1}$. Também, em muitos outros países existem instituições ou regulamentos similares, que obrigam, na maioria dos casos, a levar registros cuidadosos de todos os casos realizados e comunicar os mesmos, assim como seus resultados, à autoridade sanitária. Esta preocupação tem possibilitado conhecimento mais amplo sobre o verdadeiro impacto destas técnicas sobre a saúde da mulher, do feto e do recém-nascido. Nestes países, as clínicas devem possuir uma licença oficial de funcionamento, são visitadas pelo menos uma vez por ano por fiscais sanitários, e onde todos os registros de pacientes devem ser acessiveis e abertos para inspeção, o que tem sido motivo de críticas por parte dos diretores das clínicas $^{37}$.

No Brasil, infelizmente, as autoridades de saúde não têm implementado sistemas similares, ou até mesmo qualquer tipo de sistema minimamente organizado. Podemos assistir na mídia, de maneira geral, a profissionais médicos informando a realização de práticas muitas vezes proibidas pelos Conselhos de Medicina, nem sempre fundamentadas em verdades cientificamente comprovadas, sem que seja conhecido qualquer tipo de sanção. Da mesma forma, não existem registros nacionais destas técnicas conscientemente orga- 
nizados, e a pouca bibliografia existente limita-se a informes de casos sem valor estatístico. O Registro Latino-americano de Reprodução Assistida tenta preencher esta lacuna, mas não pode ser comparado à vigilância exercida pelas autoridades sanitárias, já que por um lado, é um modelo de registro voluntário, e por outro lado, as clínicas não estão obrigadas a abrir seus registros de pacientes, para conhecimento de resultados e complicações decorrentes.

\section{Conclusões}

Um dos problemas para comparar séries de casos, ou estudos de coorte com estes procedimentos, é a disparidade de definições entre os trabalhos, assim como os grupos utilizados como controles. Apesar de que alguns estudos tenham estratificado os casos e controles por idade e paridade, outros ignoram esta necessidade. Também em alguns trabalhos são eliminados os casos de aborto e de morte fetal intra-útero, muitos dos quais poderiam corresponder a casos de MF. Em outros que tiveram esta preocupação, a idade gestacional não permitiu analisar estes aspectos.

Aparentemente há um risco aumentado de MF em crianças nascidas por FIV 5 . Entretanto, poder-se-ia concluir que os problemas detectados nas séries revisadas são resultado das características das mulheres submetidas a estes tipos de procedimentos e que número significativo destas gravidezes são duplas ou triplas, o que eleva a prematuridade e aumenta a morbimortalidade ${ }^{38}$. Também é importante considerar que quando o tempo de tratamento para engravidar supera os três anos, independente do tratamento realizado, as chances de apresentar crianças com MF é reconhecidamente maior ${ }^{39}$. Deste modo, tem-se recomendado que estes casais sejam orientados quanto ao risco aumentado de $\mathrm{MF}$, e a realizar ultra-sonografia de alta resolução para detectar precocemente quaisquer problemas.

É prudente não negar que o número de nascidos nos últimos 25 anos, mesmo que seja substancial e em crescimento, é ainda muito pequeno em relação aos nascidos sem assistência reprodutiva e, conseqüentemente, é necessário continuar o seguimento deste tipo de nascimentos para apurar conclusões. Porém, se deve ter em conta que a associação observada de procedimentos de reprodução assistida e aumento das MF não significa relação causal. Sobretudo será necessário esperar a chegada das crianças de hoje à idade reprodutiva, especialmente aquelas nascidas por ICSI, para conhecer melhor se há problemas reprodutivos entre elas. O que se pode afirmar é que, até onde se conhece hoje, não há alterações psicomotoras nem psicológicas nestas crianças e, em relação às $\mathrm{MF}$, a última meta-análise mostrou que definitivamente há um maior número que entre as crianças concebidas naturalmente, com RR que vai de 1,4 a 2,0 (IC 95\% : 1,3-2,7) $)^{5}$ Entretanto, não há evidências em relação a um aumento do número de casos de câncer entre as crianças nascidas por procedimentos de reprodução assistida ${ }^{40-42}$. Assim, com os novos equipamentos de ultra-sonografia, seria recomendável um seguimento mais estreito destas crianças durante a gravidez.

Como o número de gravidezes múltiplas é mais alto que na população geral, levando a taxas maiores de prematuridade e BPN, isto leva a que a sociedade tenha maiores custos, alguns inerentes ao procedimento em si, a internações das mulheres mais longas e mais freqüentes e, a isto, se devem somar os gastos com unidades de cuidado intensivo destas crianças. É necessário diminuir a transferência de múltiplos embriões para reduzir ao máximo a possibilidade de gravidezes múltiplas. De qualquer forma, mais estudos serão necessários para poder elucidar estes aspectos da reprodução humana.

\section{Referências}

1. Human Fertilisation and Embriology Authority. Guide to infertility and directory of clinics, 2005-6. London: HFEA; 2005.

2. Katalinic A, Rosch C, Ludwig M; German ICSI FollowUp Study Group. Pregnancy course and outcome after intracytoplasmic sperm injection: a controlled, prospective cohort study. Fertil Steril. 2004;81(6):160416.

3. Mayer A, Lunenfeld E, Wiznitzer A, Har-Vardi I, Bentov Y, Levitas E. Increased prevalence of gestational diabetes mellitus in in vitro fertilization pregnancies inadvertently conceived during treatment with long-acting triptorelin acetate. Fertil Steril. 2005;84(3):789-92.

4. Maman E, Lunenfeld E, Levy A, Vardi H, Potashnik G. Obstetric outcome of singleton pregnancies conceived by in vitro fertilization and ovulation induction compared with those conceived spontaneously. Fertil Steril. 1998;70(2):240-5.

5. Hansen M, Bower C, Milne E, de Klerk N, Kurinczuk JJ. Assisted reproductive technologies and the risk of birth defects - a systematic review. Hum Reprod. 2005;20(2):328-38. 
6. Olivennes F, Kerbrat V, Rufat P, Blanchet V, Fanchin $\mathrm{R}$, Hazout A, et al. Follow-up of a cohort of 422 children aged from 6 to 13 and conceived by in vitro fertilization. Contracept Fertil Sex. 1997;25(5):XIIIXVIII.

7. Lancaster PA. Congenital malformations after invitro fertilisation. Lancet. 1987;2(8572):1392-3.

8. Pregnancies and births resulting from in vitro fertilization: French national registry, analysis of data 1986 to 1990. FIVNAT (French In Vitro National). Fertil Steril. 1995;64(4):746-56.

9. Westergaard HB, Johansen AM, Erb K, Andersen AN. Danish national in-vitro fertilization registry 1994 and 1995: a controlled study of births, malformations and cytogenetic findings. Hum Reprod. 1999;14(7):1896-902.

10.Bergh T, Ericson A, Hillensjo T, Nygren KG, Wennerholm UB. Deliveries and children born after in-vitro fertilisation in Sweden 1982-95: a retrospective cohort study. Lancet. 1999;354 (9190):1579-85.

11. Ericson A, Kallen B. Congenital malformations in infants born after IVF: a population-based study. Hum Reprod. 2001;16(3):504-9.

12. Martínez-Frias ML, Frias JL. VACTERL as primary, polytopic developmental field defects. Am J Med Genet. 1999;83(1):13-6.

13. Koivurova S, Hartikainen AL, Gissler M, Hemminki E, Sovio U, Jarvelin MR. Neonatal outcome and congenital malformations in children born after invitro fertilization. Hum Reprod. 2002;17(5):1391-8.

14. Friedman WF, Child JS. Congenital heart disease in the adult. In: Fauci AS, Braunwald E, Isselbacher KJ, Wilson JD, Kasper DL, Hauser SL, et al., editors. Harrison's principles of internal medicine. New York: McGraw-Hill; 1998. p. 1300-9.

15. Daniel Y, Ochshorm Y, Fait G, Geva E, Bar-Am A, Lessing JB. Analysis of 104 twin pregnancies conceived with assisted reproductive technologies and 193 spontaneously conceived twin pregnancies. Fertil Steril. 2000;74(4):683-9.

16. Minakami H, Honma Y, Matsubara S, Uchida A, Shiraishi H, Sato I. Effects of placental chorionicity on outcome in twin pregnancies: a cohort study. J Reprod Med. 1999;44 (7):595-600.

17. Stromberg B, Dahlquist G, Ericson A, Finnstrom O, Koster M, Stjernqvist K. Neurological sequelae in children born after in-vitro fertilisation: a populationbased study. Lancet. 2002;359(9305):461-5.

18. Van Loon K, Besseghir K, Eshkol A. Neural tube defects after infertility treatment: a review. Fertil Steril. 1992;58(5):875-84.

19. Silver RI, Rodriguez R, Chang TS, Gearhart JP. In vitro fertilization is associated with an increased risk of hypospadias. J Urol. 1999;161(6):1954-7.
20. Anteby I, Cohen E, Anteby E, BenEzra D. Ocular manifestations in children born after in vitro fertilization. Arch Ophthalmol. 2001;119(10):1525-9.

21. Klemetti R, Gissler M, Sevon T, Koivurova S, Ritvanen A, Hemminki E. Increasing evidence of major congenital anomalies in children born with assisted reproduction technology: what should be done? Fertil Steril. 2005;84(5):1327.

22. Olson CK, Keppler-Noreuil KM, Romitti PA, Budelier WT, Ryan G, Sparks AE, et al. In vitro fertilization is associated with an increase in major birth defects. Fertil Steril. 2005;84(5):1308-15.

23. Bonduelle $\mathrm{M}$, Legein $\mathrm{J}$, Derde MP, Buysse A, Schietecatte J, Wisanto A, et al. Comparative followup study of 130 children born after intracytoplasmatic sperm injection and 130 children born after in-vitro fertilization. Hum Reprod. 1995;10(12):3327-31.

24. Bonduelle M, Liebaers I, Deketelaere V, Derde MP, Camus M, Devroey $\mathrm{P}$, et al. Neonatal data on a cohort of 2889 infants born after ICSI (1991-1999) and of 2995 infants born after IVF (1983-1999). Hum Reprod. 2002;17(3):671-94.

25. Bonduelle M, Camus M, De Vos A, Staessen C, Tournaye H, Van Assche E, et al. Seven years of intracytoplasmic sperm injection and follow-up of 1987 subsequent children. Hum Reprod. 1999;14 Suppl 1:243-64.

26. Sutcliffe AG, Taylor B, Saunders K, Thornton S, Lieberman BA, Grudzinskas JG. Outcome in the second year of life after in-vitro fertilization by intracytoplasmic sperm injection: a UK case-control study. Lancet. 2001;357(9274):2080-4.

27. Loft A, Petersen K, Erb K, Mikkesen AL, Grinsted J, Hald F, et al. A Danish national cohort of 730 infants born after intracytoplasmic sperm injection (ICSI) 1994-1997. Hum Reprod. 1999;14(8):2143-8.

28. Sutcliffe AG, Taylor B, Grudzinskas JG, Thornton S, Lieberman B. Children conceived by intracytoplasmatic sperm injection. Lancet. 1998;352(9127):578-9.

29. Wennerholm UB, Bergh C, Hamberger L, Lundin K, Nilsson L, Wikland M, et al. Incidence of congenital malformations in children born after ICSI. Hum Reprod. 2000;15(4):944-8.

30. Meschede D, Lemcke B, Exeler JR, De Geyter C, Behre HM, Nieschlag E, et al. Chromosome abnormalities in 447 couples undergoing intracytoplasmic sperm injection: prevalence, types, sex distribution and reproductive relevance. Hum Reprod. 1998;13(3):576-82.

31. Rimm AA, Katayama AC, Diaz M, Katayama KP. A meta-analysis of controlled studies comparing major malformation rates in IVF and ICSI infants with naturally conceived children. J Assist Reprod Genet. 2004;21(12):437-43. 
32. Lie RT, Lyngstadaas A, Orstavik KH, Bakketeig LS, Jacobsen G, Tanbo T. Birth defects in children conceived by ICSI compared with children conceived by other IVF-methods; a meta-analysis. Int $\mathrm{J}$ Epidemiol. 2005;34(3):696-701.

33.Ludwig M, Katalinic A, Gross S, Sutcliffe A, Varon $\mathrm{R}$, Horsthemke B. Increased prevalence of imprinting defects in patients with Angelman syndrome born to subfertile couples. J Med Genet. 2005;42(4):289-91.

34. Olivennes F, Schneider Z, Remy V, Blanchet V, Kerbrat V, Fanchin R, et al. Perinatal outcome and follow-up of 82 children aged 1-9 years old conceived from cryopreserved embryos. Hum Reprod. 1996;11(7):1565-8.

35. Sutcliffe AG, D'Souza SW, Cadman J, Richards B, McKinlay IA, Lieberman B. Outcome in children from cryopreserved embryos. Arch Dis Child. 1995;72(4):290-3.

36. Sutcliffe AG, D'Souza SW, Cadman J, Richards B, McKinlay IA, Lieberman B. Minor congenital anomalies, major congenital malformations and development in children conceived from cryopreserved embryos. Hum Reprod. 1995;10(12):3332-7.
37. Ola B, Ledger WL. In vitro fertilisation. Curr Obstet Gynaecol. 2005;15(5):314-23.

38. High incidence of preterm births and early losses in pregnancy after in vitro fertilisation. Australian In Vitro Fertilisation Collaborative Group. Br Med J (Clin Res Ed). 1985;291(6503):1160-3.

39.Ghazi HA, Spielberger C, Kallen B. Delivery outcome after infertility- a registry study. Fertil Steril. 1991;55(4):726-32.

40.Bruinsma F, Venn A, Lancaster P, Speirs A, Healy D. Incidence of cancer in children born after invitro fertilization. Hum Reprod. 2000;15(3):604-7.

41.Klip H, Burger CW, de Kraker J, van Leeuwen FE; OMEGA-project group. Risk of cancer in the offspring of women who underwent ovarian stimulation for IVF. Hum Reprod. 2001;16(11):2451-8.

42.Lerner-Geva L, Toren A, Chetrit A, Modan B, Mandel $\mathrm{M}$, Rechavi $\mathrm{G}$, et al. The risk for cancer among children of women who underwent in vitro fertilization. Cancer. 2000;88(12):2845-7. 